\title{
Programa del asma en Atención Primaria: estudio comparativo entre dos centros de salud de Valladolid
}

\author{
S. Aníbarro Pérez, A. Alonso Clavero, M. del Nido Alonso \\ CS de Iscar. Valladolid. España.
}

Rev Pediatr Aten Primaria. 2009; I I:241-9

Ana Alonso Clavero, aloncla@amena.com

\section{Resumen}

Introducción: el asma es la enfermedad crónica infantil más prevalente en España y en el resto de países desarrollados. En Valladolid la prevalencia según los datos de la fase III del estudio ISAAC es de 7,2-9,2\%. Coincidiendo con la implantación del programa de atención al niño asmático en Castilla y León, presentamos un estudio comparativo de los resultados de dicho programa entre una población rural y otra periurbana en Valladolid.

Pacientes y métodos: estudio observacional transversal en niños de 0-14 años que cumplían criterios de inclusión en el programa de asma infantil. Se recogieron datos demográficos, pruebas alérgicas, clasificación de la gravedad, distribución estacional y tratamiento.

Resultados: las diferencias entre ambas zonas se hallaron respecto a la prevalencia (mayor en zona periurbana), sexo (70\% de varones en la zona periurbana), edad (43,7\% en la zona periurbana frente al $30 \%$ en la rural), en pruebas alérgicas (46,2\% positivas en menores de 2 años en la zona periurbana y $26 \%$ en la rural), distribución estacional (predominio invernal en la zona periurbana y primaveral en la rural en menores de 2 años) y tratamiento (en la zona rural uso de salbutamol oral, mucolíticos y antibióticos en las crisis). El resto de datos analizados son similares entre ellos y concordantes con otros estudios.

Conclusiones: al comparar las dos zonas se observan diferencias atribuibles a factores ambientales (asistencia a guarderías, infecciones, contaminación ambiental, etc.) y similitudes en una enfermedad que tiene una importante base genética, sin olvidar factores ajenos al proceso, como es la no universalización de la asistencia por pediatras a los niños de la zona rural.

Palabras clave: Asma, Niño, Estudio, Prevalencia, Alergia.

Abstract

Introduction: asthma is the more prevalent infantile chronic disease in Spain and in the rest of developed countries. According to the findings of ISAAC Phase Three, its prevalence in Valladolid is 7.2-9.2\%. Coinciding with the implementation of asthmatic children health care programme in Castilla y León, we present a comparative study based on the findings obtained by this programme between a rural and periurban population in Valladolid.

Los autores declaran no presentar conflictos de intereses en relación con la preparación y publicación de este artículo. 
Subjects and methods: a cross-sectional, observational study of children 0 to 14 years of age who satisfied inclusion criteria to the infantile asthma programme. Demographic data, allergic tests, severity classification, seasonal distribution and treatment have been collected.

Results: sex differences have been detected between both areas (70\% for male in the periurban area), age differences (43.7\% in periurban area vs. 30\% in the rural one), in allergic tests (46.2\% positives for less than 2 years of age in periurban area and $26 \%$ in the rural one) seasonal distribution differences (winter prevalence in periurban area and spring prevalence in the rural area for children less than 2 years of age) and treatment differences (use of oral salbutamol, mucolytics and antibiotics in crisis in rural area). The rest of the analysed data are similar between them and confirm the data of other studies.

Conclusions: the findings of the comparison between the two areas of study are differences due to environmental factors (day care attendance, infections, environmental pollution...) and similarities due to the fact that they are diseases with an important genetic base, not forgetting some foreign factors to the process such as the lack of universalization of pediatric assistance in the rural area.

Key words: Asthma, Children, Study, Prevalence, Allergic.

\section{Introducción}

El asma es la enfermedad crónica infantil más prevalente en España y en el resto de los países desarrollados'. La prevalencia del asma en Valladolid, según los datos de la fase III del estudio ISAAC, en pacientes de 13 a 14 años es de 7,2$9,2 \%^{2}$. La prevalencia en pacientes de 6-7 años no se estudió en Valladolid, pero se pueden tomar como referencia los datos de Pamplona (6,2-8\%), que demostraron ser semejantes a los de Valladolid en otras series.

El programa de atención al niño asmático en Atención Primaria se introduce en la cartera de servicios sanitarios de Valladolid en el año $2005^{3}$. Este programa incluye los siguientes puntos: diagnóstico (considerando como criterios de inclusión la realización de pruebas de alergia en todos los niños y de función pulmonar en los mayores de 6 años), clasificación de la gravedad según una escala reconocida, seguimiento con comprobación de la correcta realización de la técnica de inhalación utilizada y automanejo de las crisis, y plan terapéutico que incluye la evitación de desencadenantes, tratamiento farmacológico y consejo de vacunación frente a la gripe.

Presentamos un estudio comparativo de los resultados de dicho programa desde su implantación, en enero de 2005, hasta diciembre de 2006, en dos poblaciones, una rural y otra periurbana, de la provincia de Valladolid.

\section{Material y métodos}

Estudio observacional y transversal en mujeres y varones de 0 a 14 años de 
edad en dos zonas, una rural y otra periurbana de Valladolid.

La muestra estuvo formada por los niños que acudieron a la consulta en el período de tiempo de enero de 2005 a diciembre de 2006 y que cumplían los criterios de inclusión en el programa de asma infantil ${ }^{3}$. La recogida de datos se realizó mediante encuesta a los padres o tutores en la consulta de Atención Primaria y registros de las historias clínicas. Los datos recogidos fueron demográficos (sexo y edad por intervalos de 0-23 meses, 2-5 años y 6-14 años), criterios de inclusión en el programa, realización y resultados de pruebas alérgicas (test cutáneos o prick-test y determinación de IgE específica a alérgenos o Rast), clasificación de la gravedad en el momento de la revisión según escala reconocida (GI$\mathrm{NA}^{4}, \mathrm{GEMA}^{5}$ o Consenso Internacional Pediátrico $\left.[\mathrm{CIP}]^{6}\right)$, reagudizaciones estacionales, tipo de tratamiento y valoración del automanejo de las crisis asmáticas en el domicilio.

En la zona rural, con una población de 1.120 niños, 40 cumplieron los criterios de inclusión. En la zona urbana, de una población de 1.340, se incluyeron 80 pacientes. Se compararon los resultados de ambas poblaciones por rangos de edad.

\section{Resultados}

En las tablas I y II exponemos los datos demográficos de la población estudiada, así como los datos del tratamiento de los episodios asmáticos registrados.

En la zona rural fueron incluidos 40 niños. La media de edad fue de 4,9 años. La prevalencia por rangos de edad fue en menores de 2 años del $6 \%$, de 2-5 años $2,6 \%$ y en mayores de 6 años 3,2\%.

Las pruebas de alergia fueron positivas en 3 pacientes de 0-23 meses (25\%), predominando la alergia a proteínas de

Tabla I. Características demográficas de las poblaciones estudiadas

\begin{tabular}{|c|c|c|c|c|c|c|c|c|}
\hline \multirow{2}{*}{$\begin{array}{l}\text { Poblaciones estudiadas } \\
\text { Edad (años) } \\
\end{array}$} & \multicolumn{4}{|c|}{ Zona rural (población 1.120) } & \multicolumn{4}{|c|}{ Zona periurbana (población 1.340) } \\
\hline & Total & $0-1$ & $2-5$ & $6-14$ & Total & $0-1$ & $2-5$ & $6-14$ \\
\hline $\mathrm{N}$ & 40 & 12 & 14 & 14 & 80 & 35 & 19 & 26 \\
\hline Sexo $(Q / 0)$ & $19 / 21$ & $8 / 4$ & $6 / 8$ & $5 / 9$ & $24 / 56$ & $10 / 25$ & $7 / 12$ & $7 / 19$ \\
\hline Edad media (años) & 4,9 & - & - & - & 4,5 & - & - & - \\
\hline Prevalencia & - & $6 \%$ & $2,6 \%$ & $3,2 \%$ & - & $7,8 \%$ & $4,8 \%$ & $6,1 \%$ \\
\hline Pruebas alérg. positivas & 15 & 3 & 4 & 8 & 37 & 5 & 10 & 22 \\
\hline Crisis/año & - & 2,6 & 4,4 & 3,2 & - & 4 & 4 & 3 \\
\hline Crisis leves & $92 \%$ & - & - & - & $96 \%$ & - & - & - \\
\hline Predominio estacional & - & No & No & Sí & - & Sí & Sí & Sí \\
\hline
\end{tabular}


Tabla II. Características demográficas de las poblaciones estudiadas

\begin{tabular}{|c|c|c|c|c|c|c|c|c|}
\hline \multirow{2}{*}{$\begin{array}{l}\text { Poblaciones estudiadas } \\
\text { Edad (años) }\end{array}$} & \multicolumn{4}{|c|}{ Zona rural (población 1.120) } & \multicolumn{4}{|c|}{ Zona periurbana (población 1.340) } \\
\hline & Total & $0-1$ & $2-5$ & $6-14$ & Total & $0-1$ & $2-5$ & $6-14$ \\
\hline $\mathrm{N}$ & 40 & 12 & 14 & 14 & 80 & 35 & 19 & 26 \\
\hline$\beta_{2}$-agonistas inhalados & - & $58,3 \%$ & $64,2 \%$ & $100 \%$ & - & $100 \%$ & $100 \%$ & $100 \%$ \\
\hline$\beta_{2}$-agonistas orales & - & $41,6 \%$ & $35,7 \%$ & 0 & - & - & - & - \\
\hline Corticoides inhalados (CI) & - & $8,3 \%$ & $7,1 \%$ & $14,2 \%$ & - & $62,8 \%$ & $52,6 \%$ & $46 \%$ \\
\hline Corticoides orales & - & $33,3 \%$ & $14,2 \%$ & $7,1 \%$ & - & $8,5 \%$ & $5,2 \%$ & 0 \\
\hline Montelukast & - & $8,3 \%$ & $14,2 \%$ & $21,4 \%$ & - & $25,7 \%$ & $36,8 \%$ & $38,5 \%$ \\
\hline Antihistamínicos orales & - & $8,3 \%$ & $42,8 \%$ & $50 \%$ & - & $5,7 \%$ & $26,3 \%$ & $57,7 \%$ \\
\hline$\beta_{2}$-agonistas de larga & & & & & & & & \\
\hline $\begin{array}{l}\text { duración inhalados } \\
\beta_{2} \text {-agonistas de larga }\end{array}$ & 1 & 0 & 0 & 1 & 0 & 0 & 0 & 0 \\
\hline duración $+\mathrm{Cl}$ & 0 & 0 & 0 & 0 & & 0 & 0 & $23 \%$ \\
\hline Inmunoterapia & 0 & 0 & 0 & 0 & 6 & 0 & 0 & 6 \\
\hline Autocontrol & - & - & - & - & $100 \%$ & - & - & - \\
\hline
\end{tabular}

leche de vaca; en 4 pacientes de 2-5 años $(28,5 \%)$, en su mayoría positivas a gramíneas y en 8 pacientes de 6-14 años $(57,14 \%)$, fundamentalmente positivas a gramíneas, ácaros y epitelio de gato. En cuanto a la gravedad del asma, la media de crisis al año fue de 2,6 en el grupo de 0-23 meses, 4,4 en el intervalo de 2-5 años y de 3,2 en los niños de 6-14 años. La mayoría de las crisis se catalogaron como leves (92\%). La distribución estacional de los síntomas asmáticos fue: en menores de 2 años sucedieron las crisis por igual en primavera (abril y mayo) que en invierno (noviembre y diciembre), entre 2-5 años se observó idéntica distribución estacional y en el grupo de 6-14 años hubo claro predominio de crisis en meses primaverales.
Respecto al tratamiento, la totalidad de los pacientes utilizaba $\beta_{2}$-agonistas a demanda, de forma inhalada mediante el sistema Turbuhaler ${ }^{\oplus}$ o Novoli$z^{\circledR}{ }^{\circledR}$ en los mayores de 6 años; entre los menores de 2 años el 58,3\% utilizan un sistema de inhalación mediante cámara espaciadora y el resto $(41,6 \%)$ utilizan la administración por vía oral. Entre los niños de 2-5 años el 64,2\% usan cámaras de inhalación y el $35,7 \%$ la vía oral. Los corticoides inhalados se usaban en un niño de 0-23 meses $(8,3 \%)$, otro de $2-5$ años $(7,1 \%)$ y en 2 de 6-14 años (14,2\%). El uso de corticoide oral predominó en el grupo de 0-23 meses (33,3\%), siendo del $14,2 \%$ en los de $2-5$ años y de $7,1 \%$ en los de 6-14 años. 
El montelukast se administró a un niño de 0-23 meses (8,3\%), a 2 de 2-5 años $(14,2 \%)$ y a 3 de $6-14$ años $(21,4 \%)$. Los antihistamínicos orales solo se usaron en 1 niño de 0-23 meses (8,3\%), 6 de $2-5$ años $(42,8 \%$ ) y 7 de 6-14 años (50\%). Los $\beta_{2}$-agonistas de larga duración solo se usaron en un niño de 6-14 años y no se utilizaron en ningún caso las combinaciones de $\beta_{2}$-agonistas de larga duración y corticoides inhalados. La inmunoterapia se utilizó en 3 niños del grupo de 6-14 años $(21,4 \%)$ y en ningún caso en las restantes edades. En este grupo llama la atención la utilización en 2 niños de 2-5 años de mucolíticos y antibióticos como única forma de tratamiento para sus crisis asmáticas, prescritos en ambos casos por médicos de familia. El cumplimiento del tratamiento fue bueno en todos los niños, salvo en uno de 0-23 meses que tuvo problemas para el manejo de la cámara espaciadora.

En la zona periurbana se incluyeron 80 niños, con una media de edad de 4,5 años. La prevalencia fue del $7,8 \%$ en menores de 2 años, 4,8\% en pacientes de 2-5 años y del 6,1\% en mayores de 6 años. En cuanto a las pruebas de alergia, en el rango de edad de 0-23 meses fueron positivas en 5 pacientes (14,3\%), predominando la alergia a proteínas de leche de vaca y al huevo. Entre los pacientes de 2-5 años fueron positivas en 10 casos $(52,6 \%)$, sobre todo alergias ambientales (neumoalérgenos: gramíneas, ácaros, mohos y hongos). En niños de 6-14 años las pruebas fueron positivas en 22 pacientes $(84,6 \%)$, con claro predominio de los neumoalérgenos (gramíneas fundamentalmente).

Se valoró también la gravedad en forma de número de crisis al año. La media de crisis anuales fue de 3 en los mayores de 6 años y 4 en los menores de 6 años, con una distribución estacional de predominio en meses invernales en menores de 6 años (enero a marzo), y en meses de primavera en los mayores de 6 años (marzo a mayo). La mayoría (96\%) se catalogaron como leves.

Respecto al tratamiento utilizado, la totalidad de los casos empleaba $\beta_{2}$-agonistas inhalados a demanda, con cámara espaciadora en menores de 6 años y sistema Turbuhaler $^{\circledast} \mathrm{o}$ cámara espaciadora en mayores de 6 años. Los corticoides inhalados se empleaban en 22 casos de 0-23 meses (62,8\%), 10 casos de 2-5 años $(52,6 \%$ ) y 12 de $6-14$ años (46\%). El uso de corticoides orales fue en 3 pacientes de 0-23 meses (8,5\%), 1 paciente de $2-5$ años $(5,2 \%)$ y no se usó en ningún caso el rango de edad de 6-14 años. El empleo de montelukast oral fue 
en 9 casos de $0-23$ meses $(25,7 \%), 7$ casos de $2-5$ años $(36,8 \%)$ y 10 casos de 6-14 años (38,5\%).

Los antihistamínicos orales se administraron a 2 niños de $0-23$ meses $(5,7 \%)$, 5 de $2-5$ años $(26,3 \%)$ y 15 de $6-14$ años $(57,7 \%)$. El tratamiento combinado con $\beta_{2}$-agonistas de larga duración y corticoides inhalados solo se empleó en mayores de 6 años (en el 23\% de los casos) y la inmunoterapia también solo en 6 casos entre los mayores de 6 años (23\%). El autocontrol fue adecuado en el $100 \%$ de los pacientes.

\section{Discusión}

La media de edad en ambas zonas es semejante (4,5-5 años). Los datos de prevalencia en el área periurbana coinciden con los datos del estudio ISAAC (fase III) en Valladolid, mientras que los de la zona rural son claramente inferiores a partir de los 2 años. Esta diferencia puede deberse a un aumento real del asma en las zonas urbanas y periurbanas (por factores favorecedores como la contaminación, el tráfico, una mayor sensibilización alérgica, etc.) o a que los datos de dicho estudio fueron tomados en su mayoría en Valladolid capital y muy pocos en un área rural.

La distribución por sexos en la zona periurbana muestra un claro predominio de varones $(70 \%)$ en todos los rangos de edad, mientras que en la zona rural por debajo de los 2 años el $66,6 \%$ de los casos son mujeres, y en mayores de 2 años dicha proporción se invierte a favor de los varones (57,1-64,3\%). Estos datos difieren de los publicados en la mayoría de los estudios, incluido el estudio ISAAC, en el que predominan los varones en la primera infancia $(1,5: 1)^{7-10}$, igualándose en los niños mayores.

En la zona periurbana el $43,7 \%$ de los casos se encontraron entre los niños de 0 23 meses, el 23,7\% entre 2-5 años y el $32,5 \%$ entre $6-14$ años. En la zona rural el 30\% fueron niños de 0-23 meses, el $35 \%$ de 2-5 años y el resto (35\%) de 614 años. El mayor porcentaje de casos en la primera infancia en la zona periurbana puede estar relacionada con la mayor incidencia de infecciones de vías respiratorias (la infección por el virus respiratorio sincitial [VRS] es un factor de riesgo para padecer asma durante la infancia ${ }^{11,12}$ ) en las zonas urbanas y periurbanas, en las que es más habitual la asistencia a guarderías. Hay evidencias claras de la relación entre infección respiratoria y exacerbación del asma ${ }^{13}$. Las pruebas alérgicas fueron positivas en el $46,2 \%$ de los niños de zona periurbana frente al $26 \%$ en la zona rural. Sin embargo, en menores de 2 años la positividad de las pruebas alérgicas fue mayor en el área rural. En ambas 
poblaciones la frecuencia de alergia aumenta con la edad y coincide el tipo de alérgenos en cada grupo de edad (alimentarios en menores de 2 años y ambientales en mayores). Sin embargo, en nuestro estudio encontramos una incidencia de positividad de las pruebas de alergia en menores de 2 años muy superior a la encontrada en otros estudios (3$5 \%)^{14}$, mientras que la incidencia de alergia en edades escolares coincide con otras series ${ }^{15,16}$. Las diferencias entre ambas zonas coinciden con otros estudios realizados, en los que se demuestra que núcleos de población con elevados índices de contaminación inducen una mayor hiperreactividad bronquial, un deterioro de la función pulmonar y un incremento de la respuesta pulmonar a la sensibilización alérgica. Por otro lado, el tráfico podría aumentar la antigenicidad del polen y su transporte hasta el pulmón ${ }^{17}$.

La mayoría de las crisis asmáticas en ambas zonas fueron clasificadas como leves según las guías GINA ${ }^{4}, G$ MA $^{5}$ y $\mathrm{CIP}^{6}$, que respondieron bien al tratamiento en pocos días al igual que en estudios precedentes ${ }^{2,18,19}$.

Existen diferencias en la distribución estacional de las crisis entre ambas zonas en los menores de 2 años. Mientras que en la zona periurbana se concentran en los meses de invierno (relación con in- fecciones víricas y mayor asistencia a guarderías), en la zona rural predominan en los meses primaverales. En edades posteriores en ambas zonas las crisis predominan en los meses de polinización.

El tratamiento utilizado en la mayoría de las crisis es el salbutamol, de preferencia por vía inhalada (de primera elección en el tratamiento de las reagudizaciones $\left.{ }^{4-6}\right)$, aunque en la población rural se observa el uso en varios niños de salbutamol por vía oral y de mucolíticos y antibióticos, aunque estos últimos no están indicados si no hay clara evidencia de infección bacteriana ${ }^{4,20}$. Esta diferencia puede explicarse por la asistencia médica a niños por parte de otros profesionales no pediatras en la zona rural. En cuanto al resto del tratamiento no existen diferencias significativas entre ambas zonas y se acogen a las recomendaciones de las guías internacionales ${ }^{4-6}$.

En resumen, se puede decir que al comparar las dos zonas se observan diferencias que podrían ser atribuibles a factores ambientales (asistencia a guarderías, infecciones, contaminación ambiental, etc.) y similitudes en una enfermedad que tiene una importante base genética $^{21,22}$, sin olvidar los factores ajenos al proceso como es la no universalización de la asistencia por pediatras a los niños de la zona rural. 


\section{Bibliografía}

1. Oñate Vergara E, Pérez-Yarza EG, Emparanza Knön JL, Figueroa de la Paz A, Sardón Prado O, Sota Busselo I, y cols. Prevalencia actual de asma en escolares en San Sebastián. An Pediatr (Barc). 2006;64:224-8.

2. Carvajal-Urueña J, García Marcos L, Busquets-Monge R, Morales Suárez-Varela M, García de Andoin N, Batlles-Garrido J, et al. Geographic variation in the prevalence of asthma symptoms in Spanish children and adolescents. International Study of Asthma and Allergies in Childhood (ISAAC) phase III, Spain. Arch Bronconeumol. 2005; 41:659-66.

3. Borras Montaner E, Díez Astorgano A, Fernández Cuadrillero C, Fernández Pascual L, Gamarra Ortiz J, Guzmán Fernández MA, y cols. Servicio de Asma Infantil. Cartera de Servicios de Atención Primaria. SACYL; 2004.

4. GINA Report. Global Strategy for Asthma Management and Prevention, Global Initiative for Asthma (GINA) 2008 [consultado el 13/04/2009]. Disponible en www.ginasthma.org

5. Plaza Moral V, Álvarez Gutiérrez FJ, Casan Clarà $\mathrm{P}$, Cobos Barroso N, López Viña A, Llauger Rosselló MA, y cols. Guía española para el manejo del asma. Arch Bronconeumol 2003;39 (Supl 5):s3-s42.

6. Warner JO, Naspitz CK, Cropp GJA. Third International Pediatric Consensus Statement on the management of Childhood asthma. Pediatr Pulmonol. 1998;25:1-17.

7. Aguinaga I, Arnedo A, Bellido J, Guillén F, Suárez $M$. Prevalencia de síntomas relacionados con el asma en niños de 13-14 años de 9 poblaciones españolas. Grupo Español del Estudio ISAAC (International Study of Asthma and Allergies in Childhood). Med Clin (Barc). 1999;112: 171-5.
8. Grupo Regional de Trabajo sobre Asma Infantil en Atención Primaria de Asturias. Prevalencia de asma diagnosticada en la población infantil en Asturias. An Esp Pediatr. 1999;51:479-84.

9. Alba F, Flecha E, Alba J, Hernández R, Busquets $E$. Características asociadas al asma infantil en pacientes asistidos en atención primaria. Aten Primaria. 1996;18:83-6.

10. Sanz J, Martorell A, Álvarez V, Bermúdez JD, Saiz $R$, Fuentes $A$, y cols. Estudio epidemiológico de los factores de riesgo asociados con el desarrollo de patología respiratoria en la población infantil. An Esp Pediatr. 1990;32:389-98.

11. Murray M, Webb MSC, O'Callaghan C, Swarbrick As, Milner AD. Respiratory status and allergy following bronchiolitis. Arch Dis Child. 1992;62:482-7.

12. Martínez FD. Development of wheezing disorders and asthma in preschool children. Pediatrics. 2002;109:362-7.

13. Johnston SL, Pattemore PK, Sanderson G, Smith S, Lampe F, Josephs L, et al. Community study of role of viral infections in exacerbations of asthma in 9-11 year old children. BMJ. 1995;310 (6989):1225-9.

14. Busquets J. Prevalence of food allergy in asthma. Pediatr Allergy Inmunol. 1992;3: 20613.

15. Wahn U, von Mutius E. Childhood risk factors for atopy and the importance of early intervention. J Allergy Clin Inmunol. 2001;107: 56774.

16. Illi S, von Mutius E, Lau S, Nickel R, Niggemann B, Sommerfeld C, et al. The pattern of atopic sensitization is associated with the development of asthma in childhood. J Allergy Clin Inmunol. 2001; 108:709-14.

17. Salvi SS, Frew A, Holgate $S$. Is diesel exhaust a cause for increasing allergies? Clin Exp Allergy. 1999:29:4-8. 
18. Liñán S. Clasificación del asma: su utilidad. En: Cobos N (ed). Asma. Enfermedad crónica infantil. Madrid: Doyma; 1998.

19. Vermeire PA, Rabe KF, Soriano JB, Maier WC. Asthma Control and differences in management practices across seven european countries. Respir Med. 2002;96:142-9.

20. National Asthma Education and Prevention Program Expert Panel Report. Guidelines for the diagnosis and management of asthma update on selected topics 2002. J Allergy Clin Inmunol. 2002; 110(5):s180-s53.

21. Sengler C, Lau S, Wahn U, Nickel R. Interactions between genes and environmental factors in asthma and atopy: new development. Respir Res. 2002;3(1):7.

22. Holgate ST. Genetic and environmental interaction in allergy and asthma. J Allergy Clin Inmunol. 1999;104:1139-46. 